\title{
Direct connection between the different QCD orders for parton distribution and fragmentation functions
}

\author{
O. Yu. Shevchenko \\ Joint Institute for Nuclear Research
}

\begin{abstract}
The formulas directly connecting parton distribution functions (PDFs) and fragmentation functions (FFs) at the next to leading order (NLO) QCD with the same quantities at the leading order (LO) are derived. These formulas are universal, i.e. have the same form for all kinds of PDFs and FFs, differing only in the respective splitting functions entering there.
\end{abstract}

PACS: 13.85.Ni, 13.60.Hb, 13.88.+e

The extraction of PDFs and FFs from the experimental data is one of the important tasks of the modern hadron physics. The most simple and transparent way to do it is the QCD analysis of the data on measured asymmetries and cross-sections in LO QCD. One of the main advantages of such analysis is that the central values and uncertainties of measured asymmetries and cross-sections directly propagate to the central values and errors of PDFs and FFs extracted from these data in LO QCD (see, for instance, Fig. 3 in [1]). At the same time the situation with NLO analysis is much more difficult because instead of simple algebraic equations (see, for example, Eq. (2) in Ref. [1]) one deals there with complex integral equations (like, for instance, Eqs. (10)-(13) in Ref. [2]) for finding PDFs (FFs) we are interested in. The standard way to solve this problem is to apply the QCD analysis based on the fitting procedure (see [2] and references therein). However, there are unavoidable ambiguities inherent in a fitting procedure which become especially important when the quality of the fitted data is rather bad (small number of points with large errors). These are arbitrariness in the choice of the functional form (with a lot of varied parameters) of the fitted PDFs and FFs at initial scale and also ambiguities in the error band calculation (ambiguities related to the deviation of $\chi^{2}$ profile from the quadratic parabola and to the choice of $\Delta \chi^{2}$ determining the uncertainty size - see discussion on this subject in Ref. [2]). Thus, it seems to be very useful if one could obtain NLO $(\mathrm{NNLO}, \ldots)$ results on PDFs/FFs using the respective LO results as an input, without loosing, thereby, all advantages of LO analysis.

We start with some necessary notation and definitions. For the flavor non-singlet and singlet quantities we introduce the notation $\mathrm{Q}_{N S}$ and $\mathbf{V}=\left(\mathrm{Q}_{S}, \mathrm{G}\right)$, where $\mathrm{Q}_{N S}$ can be either $q_{N S}$ (nonsinglet combinations of quark densities), or $\Delta q_{N S}$ (non-singlet combinations of helicity PDFs), or combinations of transversity PDFs $\Delta_{T} q(\bar{q}) \equiv h_{1 q, \bar{q}}, \ldots$, or $D_{N S}^{h}$ ("non-singlet" combination of FFs $\left.D_{q}^{h}\right), \ldots$, while $\mathrm{Q}_{S}$ can be $q_{S}, \Delta q_{S}, D_{S}^{h}, \ldots, \mathrm{G}$ can be $g, \Delta g, D_{g}^{h}, \ldots$ In this notation the DGLAP evolution equations (see [3] for review) look as

$$
Q^{2} d \mathbf{V}\left(Q^{2}, x\right) / d Q^{2}=\left(\alpha_{s} / 2 \pi\right)\left[\mathbf{P}^{(\mathbf{0})}(x)+\left(\alpha_{s} / 2 \pi\right) \mathbf{P}^{(\mathbf{1})}(x)+O\left(\alpha_{s}^{2}\right)\right] \otimes \mathbf{V}\left(Q^{2}, x\right),
$$

\footnotetext{
${ }^{1}$ E-mail address: shev@mail.cern.ch
} 
where the convolution $(\otimes)$ is given by

$$
(A \otimes B)(x)=\int_{0}^{1} d x_{1} \int_{0}^{1} d x_{2} \delta\left(x-x_{1} x_{2}\right) A\left(x_{1}\right) B\left(x_{2}\right)=\int_{x}^{1} \frac{d y}{y} A\left(\frac{x}{y}\right) B(y),
$$

and analogously for $\mathrm{Q}_{N S}$ with the replacement $\mathbf{P}\left(x, \alpha_{s}\right) \rightarrow P\left(x, \alpha_{s}\right)=P^{(0)}(x)+\left(\alpha_{s} / 2 \pi\right) P^{(1)}(x)+$ $O\left(\alpha_{s}^{2}\right)$. Here $\mathbf{P}$ is $2 \times 2$ matrix with the elements $P_{q q}, P_{q g}, P_{g q}, P_{g g}$, and the splitting functions for unpolarized PDFs and helicity PDFs can be found in the review [4], for transversity PDFs - in the review [5], for FFs - in Ref. [6] and references therein.

Following [7] it is convenient to define the evolution operators $\mathrm{E}$ and $\mathbf{E}(2 \times 2$ matrix with the elements $\left.E_{q q}, E_{q g}, E_{g q}, E_{g g}\right)$ as

$$
\mathrm{Q}_{N S}\left(Q^{2}, x\right)=\mathrm{E}\left(\mathrm{Q}^{2}, \mathrm{x}\right) \otimes \mathrm{Q}_{N S}\left(Q_{0}^{2}, x\right), \quad \mathbf{V}\left(Q^{2}, x\right)=\mathbf{E}\left(Q^{2}, x\right) \otimes \mathbf{V}\left(Q_{0}^{2}, x\right) .
$$

Here we are interested in the initial conditions 2

$$
\mathrm{E}\left(Q^{2}=Q_{0}^{2}, x\right)=\delta(1-x), \quad \mathbf{E}\left(Q^{2}=Q_{0}^{2}, x\right)=\mathbf{1} \delta(1-x),
$$

which allow to evolve $\mathrm{Q}_{N S}$ and $\mathbf{V}$ from the initial scale $Q_{0}^{2}$ to an arbitrary scale $Q^{2}$.

It is also convenient to use, following [7], the evolution variable $t=\left(2 / \beta_{0}\right) \ln \left(\alpha_{s}\left(Q_{0}^{2}\right) / \alpha_{s}\left(Q^{2}\right)\right)$ instead of the standard variable $\ln \left(Q^{2} / \mu^{2}\right)$. Besides, we introduce the notation

$$
\left.A\right|_{L O} \equiv \hat{A},\left.\quad A\right|_{N L O} \equiv A,
$$

for any quantity $A$ at LO and NLO, respectively.

From now on we consider only the nontrivial singlet case. Transition to the simple nonsinglet case will be easily done in the end of calculations by making the replacement of the matrices with the respective commuting quantities.

In terms of quantities $t$ and $\mathbf{E}$ the DGLAP equations are rewritten in LO as

$$
\frac{d}{d t} \hat{\mathbf{E}}(\hat{t}, x)=\mathbf{P}^{(0)} \otimes \hat{\mathbf{E}}(\hat{t}, x)
$$

while in NLO they look as

$$
\frac{d}{d t} \mathbf{E}(t, x)=\left[\mathbf{P}^{(\mathbf{0})}(x)+\frac{\alpha_{s}}{2 \pi} \mathbf{R}(x)+O\left(\alpha_{s}^{2}\right)\right] \otimes \mathbf{E}(t, x)
$$

where

$$
\mathbf{R}(x) \equiv \mathbf{P}^{(\mathbf{1})}(x)-\frac{\beta_{1}}{2 \beta_{0}} \mathbf{P}^{(\mathbf{0})}(x) .
$$

Solution of (5) with the initial condition (3) $\hat{\mathbf{E}}(\hat{t}=0, x)=\mathbf{1} \delta(1-x)$ reads [7]

$$
\hat{\mathbf{E}}(\hat{t}, x)=\operatorname{Exp}\left(\mathbf{P}^{(\mathbf{0})}(x) \hat{t}\right)=\mathbf{1} \delta(1-x)+\hat{t} \mathbf{P}^{(\mathbf{0})}(x)+\frac{\hat{t}^{2}}{2 !} \mathbf{P}^{(\mathbf{0})}(x) \otimes \mathbf{P}^{(\mathbf{0})}(x)+\ldots,
$$

while to solve NLO equation (6) one can apply the elegant method of Ref. [7] based on the analogy with the perturbative quantum mechanics (see Eqs. (5.47)-(5.54) in Ref. [7]).

\footnotetext{
${ }^{2}$ We do not consider the asymptotic conditions [7] $\mathbf{E}(\mathrm{E}) \rightarrow \hat{\mathbf{E}}(\hat{\mathrm{E}})$ as $Q^{2} \rightarrow \infty$ (see Eq. (5.57) in [7]), since we deal only with particular realization (2) of the general conditions given by Eqs. (5.18) in [7].
} 
Operating in this way one obtains the general solution of (6) in the form (for a moment we omit $x$ dependence and $\delta(1-x))$

$$
\mathbf{E}(t)=\left\{\hat{\mathbf{E}}(t) \otimes\left[\mathbf{1}+\frac{\alpha_{s}\left(Q_{0}^{2}\right)}{2 \pi} \int_{t^{\prime}}^{t} d \tau \mathrm{e}^{-\beta_{0} \tau / 2} \hat{\mathbf{E}}(-\tau) \otimes \mathbf{R} \otimes \hat{\mathbf{E}}(\tau)\right] \otimes \hat{\mathbf{E}}\left(-t^{\prime}\right)\right\} \otimes \mathbf{E}\left(t^{\prime}\right) .
$$

Putting $t^{\prime} \rightarrow \infty$ in (9) one reproduces the solution (Eq. (5.54) in Ref. [7]) satisfying the boundary condition $\mathbf{E} \rightarrow \hat{\mathbf{E}}$ as $t \rightarrow \infty$. In turn, putting $t^{\prime}=0$ in (9) one gets the solution

$$
\mathbf{E}(t)=\left[\mathbf{1}+\frac{\alpha_{s}\left(Q^{2}\right)}{2 \pi} \int_{0}^{t} d \tau \mathrm{e}^{\beta_{0} \tau / 2} \hat{\mathbf{E}}(\tau) \otimes \mathbf{R} \otimes \hat{\mathbf{E}}(-\tau)\right] \otimes \hat{\mathbf{E}}(t)
$$

satisfying the boundary condition (3) we deal with.

The key point to proceed is the condition that all PDFs and FFs should take the same values in LO and NLO (as well as in NNLO,...) as $Q^{2} \rightarrow \infty$ :

$$
\mathrm{Q}_{N S}\left(Q^{2} \rightarrow \infty, x\right)=\hat{\mathrm{Q}}_{N S}\left(Q^{2} \rightarrow \infty, x\right), \quad \mathbf{V}\left(Q^{2} \rightarrow \infty, x\right)=\hat{\mathbf{V}}\left(Q^{2} \rightarrow \infty, x\right) .
$$

Though this asymptotic condition seems to be intuitively clear, let us argue it in some detail because of its great importance for what follows.

Imagine that two researchers analyse in LO (the first) and NLO (the second) the same "ideal" data - the data available with tremendous statistics even in the Bjorken "sub-limit" (so high $Q^{2}$ values are accessible that the Bjorken scaling violation becomes invisible even within extremely small uncertainties on measured asymmetries and cross-sections). For determinacy and simplicity let us suppose that they analyse the imaginary "ideal" polarized SIDIS data on pion production and extract the valence helicity PDFs $\Delta u_{V}, \Delta d_{V}$ from the proton and deuteron difference asymmetries (see Ref. [8] and references therein) measured in the Bjorken "sub-limit". The first uses LO formulas $A_{p}^{\pi^{+}-\pi^{-}} \sim\left(4 \Delta u_{V}-\Delta d_{V}\right) /\left(4 u_{V}-d_{V}\right)$ and $A_{d}^{\pi^{+}-\pi^{-}} \sim$ $\left(\Delta u_{V}+\Delta d_{V}\right) /\left(u_{V}+d_{V}\right)$ (i.e., performs the analysis analogous to one of COMPASS [9]), and the second their NLO generalization (Eqs. (6-10) in Ref. [8]). Besides, for self-consistency, both imaginary researches do not use the existing parametrizations on $u_{V}, d_{V}$ but extract these quantities themselves (as well as the integrated over cut in $z$ differenc $3 D_{1}-D_{2}$ of favored and unfavored pion FFs) using the same SIDIS data on pion production averaged over spin and studying the quantities $F_{2 p\left(d,{ }^{3} \mathrm{He}, \ldots\right)}^{\pi^{+}}-F_{2 p\left(d,{ }^{3} \mathrm{He}, \ldots\right)}^{\pi^{-}}$, where in both LO and NLO only $u_{V}, d_{V}$ and $D_{1}-D_{2}$ survive. It is obvious that all terms with convolutions $\otimes$ (see Eqs. (6-10) in Ref. [8]) distinguishing NLO and LO equations for finding $\Delta u_{V}, \Delta d_{V}$ and $u_{V}, d_{V}, D_{1}-D_{2}$ just disappear as one approaches the Bjorken limit, so that comparing the results on these quantities obtained in the Bjorken "sub-limit" both researchers could not discriminate between them.

So, let us pass to limit $Q_{0}^{2} \rightarrow \infty$ in Eq. (2) using the asymptotic condition (11). Then, on the one hand (NLO evolution)

$$
\mathbf{V}\left(Q^{2}, x\right)=\mathbf{E}(t \rightarrow-\infty, x) \otimes \mathbf{V}\left(Q_{0}^{2} \rightarrow \infty, x\right)=\mathbf{E}(t \rightarrow-\infty, x) \otimes \hat{\mathbf{V}}\left(Q_{0}^{2} \rightarrow \infty, x\right),
$$

and, on the other hand (inverse LO evolution)

$$
\hat{\mathbf{V}}\left(Q_{0}^{2} \rightarrow \infty, x\right)=\hat{\mathbf{E}}(\hat{t} \rightarrow \infty, x) \otimes \hat{\mathbf{V}}\left(Q^{2}, x\right) .
$$

\footnotetext{
${ }^{3}$ On simultaneous determination of valence PDFs and $D_{1}-D_{2}$ from the SIDIS data see, for example, [10].
} 
Combining Eqs. (12) and (13) one obtains

$$
\mathbf{V}\left(Q^{2}, x\right)=\left[\lim _{Q_{0}^{2} \rightarrow \infty} \mathbf{E}(t, x) \otimes \hat{\mathbf{E}}(-\hat{t}, x)\right] \otimes \hat{\mathbf{V}}\left(Q^{2}, x\right) .
$$

Using Eqs. (8), (10) and the relation $\lim _{Q^{2} \rightarrow \infty}\left(\alpha_{s} / \hat{\alpha}_{s}\right)=1$ we arrive at the connection formula between NLO and LO flavour singlet PDFs (FFs) $\mathbf{V}$ and $\hat{\mathbf{V}}$ at the same finite $Q^{2}$ value

$$
\begin{gathered}
\mathbf{V}\left(Q^{2}, x\right)=\left[\mathbf{1} \delta(1-x)-\frac{\alpha_{s}\left(Q^{2}\right)}{2 \pi} \int_{-\infty}^{0} d \tau \mathrm{e}^{\beta_{0} \tau / 2} \hat{\mathbf{E}}(\tau, x) \otimes \mathbf{R}(x) \otimes \hat{\mathbf{E}}(-\tau, x)\right] \\
\otimes \operatorname{Exp}\left(-\frac{2}{\beta_{0}} \ln \frac{\alpha_{s}\left(Q^{2}\right)}{\hat{\alpha}_{s}\left(Q^{2}\right)} \mathbf{P}^{(\mathbf{0})}(x)\right) \otimes \hat{\mathbf{V}}\left(Q^{2}, x\right)
\end{gathered}
$$

where all dependence on the unreachable infinite point $Q_{0}^{2}$ just cancels out.

In the non-singlet case the relation (15) is significantly simplified. The terms $\hat{E}(\tau, x) \equiv$ $\operatorname{Exp}\left(\tau P^{(0)}(x)\right)$ and $\hat{E}(-\tau, x)$ cancel out each other in the integrand and one easily obtains

$$
\begin{gathered}
\mathrm{Q}_{N S}\left(Q^{2}, x\right)=\left[\delta(1-x)+\frac{\alpha_{s}\left(Q^{2}\right)}{2 \pi}\left(\frac{\beta_{1}}{\beta_{0}^{2}} P^{(0)}(x)-\frac{2}{\beta_{0}} P^{(1)}(x)\right)\right] \\
\otimes \operatorname{Exp}\left(-\frac{2}{\beta_{0}} \ln \frac{\alpha_{s}\left(Q^{2}\right)}{\hat{\alpha}_{s}\left(Q^{2}\right)} P^{(0)}(x)\right) \otimes \hat{\mathrm{Q}}_{N S}\left(Q^{2}, x\right) .
\end{gathered}
$$

Eqs. (15) and (16) connecting flavour singlet and non-singlet quantities in NLO with the same quantities in LO is the main result of the paper. Let us briefly discuss their practical use.

There are not any problems with application of Eq. (16) and the task of reconstruction of NLO non-singlet quantities from LO ones is reduced just to the trivial calculation of the integrals entering the convolutions $\otimes$. Indeed, the parameter $\epsilon \equiv-\left(2 / \beta_{0}\right) \ln \left(\alpha_{s} / \hat{\alpha}_{s}\right)$ is very small even at the minimal (the lower boundary of the experimental cut on $Q^{2}$ is usually about $1 \mathrm{GeV}^{2}$ ) really available $Q^{2}$ values, so that one can achieve very good accuracy keeping only few first terms in the expansion $\operatorname{Exp}\left(\epsilon P^{(0)}(x)\right)=\delta(1-x)+\epsilon P^{(0)}(x)+\left(\epsilon^{2} / 2 !\right) P^{(0)}(x) \otimes P^{(0)}(x)+\ldots$ Certainly, the same statement holds for term $\operatorname{Exp}\left(\epsilon \mathbf{P}^{(\mathbf{0})}(x)\right)$ in Eq. (15), but there arises an additional problem how to deal with the integral over $\tau$. As usual, the problem is easily solved in the space of Mellin moments. Notice that $Q^{2}$ independent integral over $\tau$ in Eq. (15) just coincides with the quantity $-U(x)$ in Ref. [7] (see Eq. (5.45) in [7]), which enters the solution of DGLAP with the boundary conditions $\lim _{Q^{2} \rightarrow \infty} \mathbf{E}(\mathrm{E})=\hat{\mathbf{E}}(\hat{\mathrm{E}})$ (see footnote 2 ). Then, applying the inverse Mellin transformation, one easily obtains instead of (15) the formula suitable 5 for numerical calculations

$$
\mathbf{V}\left(Q^{2}, x\right)=\left[\mathbf{1} \delta(1-x)+\frac{\alpha_{s}\left(Q^{2}\right)}{2 \pi} \int_{C-i \infty}^{C+i \infty} d n \frac{x^{-n}}{2 \pi i} U(n)\right] \otimes \operatorname{Exp}\left(\epsilon\left(Q^{2}\right) \mathbf{P}^{(\mathbf{0})}(x)\right) \otimes \hat{\mathbf{V}}\left(Q^{2}, x\right)
$$

where $2 \times 2$ matrix $U(n) \equiv \int_{0}^{1} d x x^{n-1} U(x)$ is given by Eq. (5.41) in Ref. [7].

In summary, the formulas allowing to transform LO parton distribution and fragmentation functions to NLO ones are derived. To obtain these formulas we use as an input only the DGLAP evolution equations and the asymptotic condition that PDFs (FFs) at different QCD orders become the same in the Bjorken limit. Due to universality of this input the connection

\footnotetext{
${ }^{4}$ Using Eq. (5.28) in Ref. [7] for $U$ and the obvious relation $Q^{2} d\left[\operatorname{Exp}\left(\left(2 / \beta_{0}\right) \ln \hat{\alpha}_{s} \mathbf{P}^{(\mathbf{0})}\right) \otimes \hat{\mathbf{V}}\right] / d Q^{2}=0$ one can immediately check that r.h.s. of Eq. (15) indeed satisfies the NLO DGLAP equation (11).

${ }^{5}$ In Ref. [1] one can find the efficient algorithm for the numerical calculation of the integral over $n$ (proper choice of the integration contour, etc. - see discussion around Eq. (3.2) in Ref. [11]).
} 
formulas are also universal, i.e. they are valid for any kind of PDFs (FFs) we deal with. Besides, it is obvious that operating in the same way one can also establish the connection of PDFs (FFs) at LO (as well as at NLO) with these quantities at any higher QCD order (NNLO, NNNLO, ...), and the only restriction here is the knowledge of the respective splitting functions.

\section{References}

[1] M. Alekseev et al. [COMPASS Collaboration], Phys. Lett. B 693 (2010) 227.

[2] A. N. Sissakian, O. Y. Shevchenko and O. N. Ivanov, Eur. Phys. J. C 65 (2010) 413.

[3] G. Altarelli, Phys. Rept. 81 (1982) 1.

[4] B. Lampe and E. Reya, Phys. Rept. 332 (2000) 1.

[5] V. Barone, A. Drago and P. G. Ratcliffe, Phys. Rept. 359 (2002) 1.

[6] P. Nason and B. R. Webber, Nucl. Phys. B 421 (1994) 473.

[7] V. Furmanski and R. Petronzio, Z. Phys. C 11 (1982) 293.

[8] O. Y. Shevchenko, R. R. Akhunzyanov and V. Y. Lavrentyev, Eur. Phys. J. C 71 (2011) 1713.

[9] M. Alekseev et al. [COMPASS Collaboration], Phys. Lett. B 660 (2008) 458.

[10] M. Arneodo et al. [European Muon Collaboration], Nucl. Phys. B 321 (1989) 541.

[11] A. Vogt, Comput. Phys. Commun. 170 (2005) 65 (hep-ph/0408244). 